\title{
A ROLE OF SALMON SOSC1 IN ANTIVIRAL IMMUNITY?
}

\section{Mehrdad Sobhkhez, Lisbeth Joensen, Linn G. Tollersrud, Hanna Thim \& Jorunn B. Jørgensen ${ }^{\S}$}

The Norwegian College of Fishery Science, UiT The Arctic University of Norway, N-9037 Troms $\phi$, Norway

Suppressors of cytokine signaling (SOCS) proteins are intracellular proteins that inhibit cytokine signaling in a variety of cell types. Several SOCS family members have been identified in bony fish. The most well-described function of SOCS proteins is the negative regulation of the JAK/STAT pathway. Our group has identified several Atlantic salmon SOCS genes, including a SOCS1 homolog that demonstrated a strong negative regulatory activity on type I and type II IFN signaling in salmonid cell-lines, while the salmon SOCS2a and b and CISH only moderately affected IFN responses (Skjesol et al 2014). Additionally stimulation of splenocytes with the RNA mimick poly I:C and CpG DNA induced considerably higher SOSC1 transcript levels compared to SOCS2 and CISH levels. Together the data suggest that SOCS1 has a particular role in the control of viral infections, which also holds true in mammals. In this study, we were interested finding out whether two important salmon pathogenic RNA viruses, salmon alphavirus (SAV) or infectious pancreas virus, were able to modulate SOCS expression in salmon cells lines. We also tested SOCS expression in SAV challenged Atlantic salmon. Furthermore, we asked the questions whether the negative impact of the salmon SOCS1 on IFN signaling would affect virus replication and the expression of ISGs. As important tools for these studies, CHSE-cells overexpressing SOCS1 or SOCS2 were developed and used for both viral infection studies and to measure ISG expression upon stimulation by IFNs. Results for these experiments will be presented and discussed.

Keywords. SOCS, JAK/STAT signaling, interferons, Atlantic salmon, virus

§Corresponding author. Tel.: +4795095754.

E-mail address: jorunn.jorgensen@uit.no

Skjesol A, Liebe T, Iliev DB, Thomassen E, Greiner-Tollersrud L, Sobhkhez M, Lindenskov Joensen L, Secombes CJ \& JB Jørgensen (2014). Functional conservation of Suppressors of Cytokine Signalling proteins between teleosts and mammals: Atlantic salmon SOCS1 binds to STAT1 and supress type I and II IFN signalling.

Developmental \& Comparative Immunology.45:177-189. 